RUNNING HEAD: Emotion components and social relations

\title{
Emotion Components and Social Relations
}

\author{
Brian Parkinson \\ Department of Experimental Psychology \\ University of Oxford \\ Tinbergen Building \\ 9 South Parks Road \\ Oxford, OX1 3UD, UK
}

Email: brian.parkinson@psy.ox.ac.uk

Work on this commentary was supported by the Economic and Social Research Council (ESRC), UK grant ES/L016486/1, "Communicating Appraisals and Social Motives (CASM)." 


\section{Emotion Components and Social Relations}

The history of classic emotion theories can be seen as a series of attempts to identify the specific causal process that generates the phenomenon in question: the "goose which lays the golden eggs" in James' (1898, p. 449) terms. For example, successive explanations have focused on perceptions of bodily feedback (e.g., James, 1898), activation of specialized brain regions or circuits (e.g., Cannon, 1929), appraisals of personal significance (e.g., Arnold, 1960), or selfattribution of body states (e.g., Schachter, 1964). Their common presupposition is that emotion is a distinct and distinctive phenomenon requiring explanation in terms of a correspondingly specific process or set of processes. Determining what makes emotion emotional apparently necessitates the identification of some transformatory ingredient that achieves that alchemical process (Parkinson, 2013). But what if there are no golden eggs for the goose to lay? What if the phenomenon that theorists are trying to explain is not unitary, intact, or scientifically tractable in the first place? Would that make the search for special causes a wild goose chase?

In a parallel but more sporadic history, skeptical theorists have questioned whether emotion really is a coherent and distinctive entity after all (see Griffiths, 1997). For example, Duffy (1941) argued that everything supposedly "emotional" is in fact some combination of direction and activation parameters, just like other aspects of human functioning. More recently, Russell (1980) proposed that most of the phenomena constructed by perceivers as emotions can be specified by reference to the affective dimensions of pleasure and arousal. According to these views, what needs explaining is not emotion per se but rather the various states, processes, and episodes that laypeople represent in emotional terms (see also Mandler, 1975; Parkinson, 1995).

Moors' (this issue) integrative target article combines insights from both classic and skeptical theorists. Her conclusion is that emotion is not a golden egg, but that goose-hunting is not wildly misguided, because it may lead researchers to causal mechanisms that can account for 
processes conventionally associated with emotion, and for associations between these supposed emotion components.

In reaching these conclusions, Moors balances tight logical reasoning with deep and expansive knowledge of emotion theories and philosophy of science. She convincingly integrates two skeptical theories that are usually seen as unrelated, and thereby constructs an account that advances on either of its direct predecessors in important ways. In many respects, Moors' proposed research program represents the state of the art in cognitive-behavioral emotion theory. It uses some of the most sophisticated conceptual resources currently available to address questions that have dominated psychological emotion research since the time of William James.

But are these the only or most important questions? Do the proposed answers apply to the full range of phenomena investigated by the various disciplines and subdisciplines of contemporary emotion research? Or are some of those phenomena simply outside the program?

This commentary focuses on two aspects of Moors' analysis. First, I consider her positive proposals about the role of appraisals and goal-directed mechanisms in the causation of emotion-related behaviour, and argue that these processes are more fully understood in their broader temporal and social context. Attention to the functional properties of emotional behavior from this wider perspective may reveal an underlying coherence that is less apparent at the molecular level. Next, I turn to the skeptical aspect of Moors' proposal, and argue that vernacular emotion concepts may play constitutive roles in the perception, experience, and regulation of emotional activity. These representation-dependent processes extend the range of possible ways in which emotions can function to align relations between people and events in the shared environment, and add another layer of meaningful structure to emotional processes. 


\section{Delimited and Extended Appraisal}

Classic appraisal theories (e.g., Arnold, 1960; Lazarus, 1966; 1991) sought to explain coherent patterns of response spanning cognitive, physiological, experiential, expressive and motivational components by reference to processes designed to detect the adaptive relevance of objects, events or transactions. From their perspective, the mental system knows when to output a co-ordinated emotion on the basis of appraised relational meaning. Further, this meaning represents an integrated perception or interpretation of the current situation's relevance for personal well-being (e.g., a core relational theme, Lazarus, 1991). For example, people get angry because they have concluded (consciously or unconsciously) that someone else is to blame for some bad thing that has happened. Similarly, people become afraid because they are uncertain about their ability to cope with an anticipated event. In both cases, the emotion involves an orchestrated preparation of the body and mind to deal with the concern identified by the appraisal. Moors (this issue) cogently sets out some of the theoretical and empirical challenges facing such accounts, before presenting her alternative approach, which seeks to explain specific emotion-related behaviors rather than integrated emotion syndromes, and extends the range of cognitive operations that generate these components to include goal-based mechanisms.

Such an approach neatly sidesteps some of the conceptual and empirical problems faced by classic appraisal theories. For example, explaining fear in terms of the detected frightening qualities of the current transaction implied a troubling overlap between cause (appraisal) and effect (emotion), especially when a key component of the effect is precisely the sense that what is happening is frightening (Lazarus, 1991). Moors' (this issue) analysis resolves this issue in two interrelated ways. First it draws on molecular appraisal or goal representations rather than 
molar relational meanings (such as danger or other-blame) that potentially overlap with integrated emotional responses. Second, it focuses explanation not on a co-ordinated and personally meaningful emotion syndrome but on specific "emotional" behaviors that are more easily separable from prior cognitive operations. Relations between those behaviors and other components (e.g., conscious experience) may then be explained by separate principles.

A related limitation of classic appraisal theories arises from their focus on proximal causes. Emotions are seen as depending on integrated appraisals of personal significance, but once these appraisals are integrated, much of the causal process is already complete (Parkinson, 1995). Any holistic appraisal starts to seem more like an already developing emotion than a separable causal step preceding and determining the output of that emotion (Parkinson, 1997; 2001). Once a person has begun to see things as frightening, they are usually already frightened (Dewey, 1894), begging the question of what caused them to see things as frightening in the first place. In short, the classic appraisal approach pays too little attention to the factors that lead to final conclusions about personal significance. Specifying these factors in detail may leave little further explanatory work for appraisal to do.

Moors' (this issue) approach again helps to resolve this problem by focusing on molecular rather than molar information-processing as explanans, and behavioral components rather than integrated emotions as explananda. Thus, there is no need for an alchemical relational theme to convert the base metal of cold informational input to the gold of a coordinated emotion syndrome (Lazarus \& Smith, 1988). However, Moors’ main explanatory focus still falls on a single instant when representations trigger emotion-relevant output without specifying in detail how those representations are generated. For example, goal-directed mechanisms depend on comparisons of expectancies and values associated with action options, 
so explaining their operation requires specification not only of how action options are initially selected for comparison, but also of how associated expectancies are computed and evaluated. Clearly, a range of possible factors feed into this process, providing more distal explanations of the specific emotion-related behavior in question.

To address these issues, Moors acknowledges that the immediate causal process is embedded in hierarchical cybernetic systems, which set the parameters for molecular appraisals and goal-based representations. These processes help to establish low-level goals and values on the basis of top-down checks of progress towards more abstract or inclusive goals. A potential problem with this theoretical move is that it may reintroduce an integrative emotion-generating process at a higher level, thus undermining Moors' skepticism about emotional coherence (and potentially bringing back many of the conceptual issues that the lower-level focus was intended to avoid).

For many emotion theorists, the higher-level processes that determine the priority or control precedence of concerns are of more direct interest than the immediate stimulus-focused ones. For example, Frijda (1986) characterizes emotions as modes of action readiness that are oriented to abstractly specified concerns rather than specific goal-directed behaviors. In particular, "anger" is about removing goal blockages, but not about specific movements such as pushing or punching that might help achieve this "e-motivational” goal (Roseman, Wiest, \& Swartz, 1994). Thus, angry people are oriented to whatever aspects of the current transaction are potential barriers to ongoing projects and adjust their readiness for relational activity accordingly. Moors' focus falls on how the body and mind prepare for specific immediate responses when exposed to particular stimulus constellations, and not on how the priority of different concerns changes over more extended episodes. However, functional commonalities 
between superficially dissimilar behaviors (e.g., shouting or punching to remove a goal blockage) may only become apparent when considering the higher-level goals that motivate them. And it is at this functional level that "emotion" may start to seem like a more coherent and scientifically useful concept. Indeed, the concern-directed orientation of the action readiness mode may provide a basis for explaining both the intentional aspect of first-order emotional experience and the regulation of emotional behavior. According to Frijda (2005, p. 474), "such emotion experience is best characterised as a perception of a meaningful world that is filled with calls for action.”

Moors' focus on goal-directed practical action as explanandum also tends to marginalize any communicative functions of emotional expression and behavior. One of the important features of action readiness modes as forms of embodied relational activity is that they signal possible lines of future action to other individuals (e.g., Mead, 1934). For example, our apparent readiness to push against the goal blockages presented by other people may discourage those people from continuing to block our goals. Further, our perceptible orientation to unfolding events responds on-line to interpersonal feedback provided by the other person's orientation, thereby producing a co-regulated system of relation alignment out of which emotional structure may emerge (Parkinson, 2008).

Understanding these social dynamics is outside the scope of Moors' (this issue) deliberately delimited analysis, but still sets an important context for the operation of the more specific, low-level, and momentary processes. Indeed, socially distributed control systems may help to co-ordinate and give meaning to specific emotional behaviors (e.g., Lewis, 1996; Parkinson, 2001). Attention to broader interpersonal (and group-based) processes can thus 
provide answers to certain kinds of scientific question about emotion causation prior to and independent of any detailed specification of localised subprocesses.

In this section, I have argued that specific momentary behaviors may achieve emotional significance when contextualised in temporally extended and socially distributed patterns of relational activity. Thus, focusing on specific momentary causes risks missing a bigger picture that may reveal a more abstract level of emotional structure. In the next section, I consider whether laypeople's application of categorical emotion representations to relational activity can add a further layer of consolidation.

\section{Representation and Reality}

In developing her rationale for focusing explanation on emotion-related components rather than integrated emotional syndromes, Moors (this issue) endorses the skeptical view that the vernacular concept of "emotion" is unsuitable for scientific purposes. I agree with her reading of the limited evidence for structural coherence, and concur with the conclusion that English-language emotion concepts do not carve nature cleanly at its joints. However, I also believe that studying the way emotional concepts are deployed by laypeople in different societies has direct scientific relevance to our understanding of how emotion components operate (Parkinson, 1995). In this section, I therefore set out some of the ways in which emotion representations might impact on "emotional" reality.

When considering the scientific utility of "emotion" representations, Moors draws an analogy with the concept of "air." Centuries ago, researchers concluded that air was a mixture of different gases rather than a single compound with determinate structure. Hence, contemporary chemists no longer treat air as a phenomenon requiring unitary explanation. However, this does not mean that the concept of air no longer serves any role in other scientific contexts such as 
meteorology, geology or aeronautics. Similarly, emotion's apparent lack of structural integrity may make the concept less useful for certain kinds of scientific analysis than others.

The application of the concept of "air" to the phenomenon it represents makes no difference to that phenomenon. By contrast, people's representation of their own or other people's conduct in emotional terms can directly affect processes that shape that conduct. For example, thinking of yourself in angry terms may focus your attention on angering aspects of the situation, and thinking of someone else as angry may lead you to act more defensively thus eliciting reciprocal effects on their behaviour. More generally, emotion concepts provide a shared set of interpretations and evaluations that bring real consequences for how "emotional" action plays out in the social arena. Based on observations such as this, some theorists have concluded that emotional phenomena are at least partly constituted by the application of emotion representations. In a sense, believing in emotion may become a kind of self-fulfilling prophesy (e.g., Snyder, 1984).

For example, Schachter (1964) argued that labelling oneself as "angry" or "euphoric" when experiencing physiological arousal in an appropriately "emotional" context was sufficient to produce an emotional state. Drawing an emotional conclusion about one's experience thus made that experience emotional. Barrett's (e.g., 2006; Barrett, Wilson-Mendenhall, \& Barsalou, 2015) conceptual act theory provides a more sophisticated account of how such a process might operate. In her view, emotion categories are embodied simulations which play a role in predictive coding. Conceptualizing experience as angry, for example, changes your orientation to what is happening inside and outside the body, channelling perception and response along trajectories specified by the mental script for angry episodes. According to both these accounts, 
“emotion" becomes a more coherent and more scientifically relevant concept precisely because it is deployed in self-perception.

Self-perception accounts tend to downplay the ontogenetic priority of other people's emotion representation over reflexive emotion representation. In fact, children only learn to see experience and behavior in emotional terms after their caregivers have made the relevant cultural categories and scripts available to them. Further, caregivers' application of emotion representations to children's actions makes a difference to how those actions unfold. For example, parents helping a child who is showing apparent distress while trying to push a square block into a round hole may adopt an empathetic "cross" expression and guide the child toward a more appropriately shaped aperture. Over time, the child may come to associate a stylised emotion display with a contextualised desire, and may even learn that adopting a corresponding display can recruit other people to help fulfil that desire. In short, because parents attribute emotions to their children, children may end up taking relational positions that match those attributed emotions.

As a result of their early emotional interactions with parents, children ultimately learn to communicate symbolically using nonverbal representations of emotions (Holodynski \& Friedlmeier, 2006). In the right context, narrowed eyes and tightened lips can convey an articulated trajectory of action, and thereby encourage others to adopt or contest the orientations towards current circumstances specified by the shared script for anger episodes. For example, an "angry" expression directed at another person may encourage onlookers to see that other person as afraid (Mumenthaler \& Sander, 2012). Similarly, an anxious expression may lead others to be act in a more risk averse manner (e.g., Parkinson, Phiri, \& Simons, 2012; Parkinson \& Simons, 2009; Sorce, Emde, Campos, \& Klinnert, 1985). Script-informed emotion presentations thus 
provide a means of directing interpersonal attention and communicating object-directed appraisals, thereby influencing emotional behavior. Correspondingly, someone else's interpretation and evaluation of your behaviour along scripted lines may influence their expectations, appraisals and actions, leading to further reciprocal effects on your own orientation to, and mode of engagement with, unfolding events.

Of course, emotion concepts are not invented afresh by every person who applies them either to their own behavior or to the behavior of another person. Instead, they are outcomes of a process of cultural evolution that is shaped by ideological forces as well as the empirical characteristics of the represented phenomena themselves (which in any case are transformed by application of the evolving representations). According to Averill's (1980) social constructionist theory, emotion scripts serve the specific cultural function of resolving tensions between competing social norms. They not only specify transitory social roles shaping the emotional behavior of socialised agents but also allow these agents to disown responsibility for any counter-normative behavior by formulating their responses as passions rather than actions. I shout at you because my socialized desire for fairness means that I cannot let what you did stand unopposed, but my shouting is "angry" shouting because to attack you deliberately would be unacceptable in my society (Averill, 1982). The angry role thus allows me to suspend one set of social norms while following another, and to do so without being considered responsible for this temporary suspension of norms.

Averill's theory suggests that emotion representations can directly affect the enactment as well as the perception and interpretation of behavior. If so, cultural rules about how people should conduct themselves under specific "emotional" circumstances add another layer of coherence to behavior that is represented in emotional terms. 
This section has argued that categorical emotion representations specify scripts for emotional episodes and therefore cannot be ignored when formulating a comprehensive explanation of emotional behavior. However, this point does not undermine Moors' more general skepticism about the scientific validity of vernacular emotion concepts. For one thing, many recognisably "emotional" episodes are not influenced by the application of emotion representations. The actors involved are oriented instead to functional concerns that have no necessary or intrinsic relations to emotion representations. These functional concerns can operate independently of any characterization of experience or behavior in emotional terms. For example, infants are not enacting bashful or embarrassed roles when they turn their heads away from extending eye contact and break into a "coy" smile (Reddy, 2000). Instead, they are directly regulating their interpersonal contact with interaction partners. The response of those interaction partners in turn does not only depend on seeing the infant's behavior as coy but also reflects more direct adjustments to a withdrawal of interpersonal attention.

Even when emotion representations play a more direct role in shaping behavior, other factors continue to exert separable influences. The resulting response patterns are therefore unlikely to correspond cleanly to the interpretative templates specified by our shared cultural histories. For example, when I pull an angry face to show that I see your behavior as insulting, other muscles are also adjusting to address the specific form of insult, pushing against any direct obstacle or squirming forcibly to break free from any tightening corner. Both of the latter responses are readable as “anger” but are not caused by anybody's representation of events in angry terms. In other words, emotion representations contribute to the functional integrity of emotion episodes but rarely if ever fully determine that integrity. 


\section{Conclusions}

This commentary has argued that emotional processes derive coherence from at least two sources (see also Parkinson, 2009). First, relational concerns provide a functional basis for emotional behavior and shape the prioritization and reprioritization of more local contextualised goals, including communicative and social influence goals. Second, cultural representations with specifically emotional meanings provide a shared currency for scripted or communicatively oriented interactions. These two processes interact because relational functions may be addressed by following emotion scripts or by orienting others to those scripts. Further, both kinds of regulatory process may be distributed between individuals and consolidate as a process of interpersonal negotiation. Attention to momentary intrapsychic processes as mediators of their effects on emotional behavior may be necessary at some levels of analysis but not others. Moors' otherwise integrative analysis is not designed or equipped to deal with all the interpersonal processes of relation alignment outlined above. Although her outlined research program is valuable and potentially productive, it clearly cannot address the full range of phenomena that are currently of interest to emotion researchers. A broader integration needs to acknowledge emotion's communicative functions, and the transformative effects of culturally socialised emotion scripts on the production and perception of verbal and nonverbal emotional actions. Emotional phenomena are shaped by interpersonal processes as well as singlemindedly intrapsychic ones. They are oriented to abstract concerns and long-term projects as well as immediate contextualised goals. 


\section{References}

Arnold, M. B. (1960). Emotion and personality (Volume 1): Psychological aspects. New York: Columbia University Press.

Averill, J. R. (1980). A constructivist view of emotion. In R. Plutchik \& H. Kellerman (Eds.), Theories of emotion (pp. 305-340). New York: Academic Press.

Averill, J. R. (1982). Anger and aggression: An essay on emotion. New York: Springer.

Barrett, L. F. (2006). Solving the emotion paradox: Categorization and the experience of emotion. Personality and Social Psychology Review, 10, 20-46.

Barrett, L. F., Wilson-Mendenhall, C. D., \& Barsalou, L. W. (2015). The Conceptual Act Theory: A roadmap. In L. F. Barrett \& J. A. Russell (Eds.), The psychological construction of emotion (pp. 83-110. New York: The Guilford Press.

Dewey, J. (1894). The theory of emotion I: Emotional attitudes. Psychological Review, 1, 553569.

Duffy, E. (1941). An explanation of "emotional" phenomena without the use of the concept "emotion." Journal of General Psychology, 25, 283-293.

Frijda, N. H. (1986). The emotions. Cambridge: Cambridge University Press.

Frijda, N. H. (2005). Emotion experience. Cognition and Emotion, 19, 473-497.

Griffiths, P. E. (1997). What emotions really are. Chicago, IL: University of Chicago Press.

Holodynski, M., \& Friedlmeier, W. (2006). Development of emotions and emotion regulation. New York: Springer.

Lazarus, R. S. (1966). Psychological stress and the coping process. New York: McGraw-Hill. Lazarus, R. S. (1991). Emotion and adaptation. New York: Oxford University Press 
Lazarus, R. S., \& Smith, C. A. (1988). Knowledge and appraisal in the cognition-emotion relationship. Cognition and Emotion, 2, 281-300.

Lewis, M. D. (1996). Self-organizing cognitive appraisals. Cognition and Emotion, 10, 1-15.

Mandler, G. (1975). Mind and emotion. New York: Wiley.

Mead, G. H. (1934). Mind, self, and society. Chicago, IL: Chicago University Press.

Moors, A. (this issue). Integration of two skeptical emotion theories: Dimensional appraisal theory and Russell's psychological construction theory. Psychological Inquiry.

Mumenthaler, C., \& Sander, D. (2012). Social appraisal influences recognition of emotions. Journal of Personality and Social Psychology, 102, 1118-1135.

Parkinson, B. (1995). Ideas and realities of emotion. London: Routledge.

Parkinson, B. (1997). Untangling the appraisal-emotion connection. Personality and Social Psychology Review, 1, 62-79.

Parkinson, B. (2001). Putting appraisal in context. In K. R. Scherer, A. Schorr, \& T. Johnstone (Eds.), Appraisal processes in emotion: Theory, methods, research (pp. 173-186). New York: Oxford University Press.

Parkinson, B. (2008). Emotions in direct and remote social interaction: Getting through the spaces between us. Computers in Human Behavior, 24, 1510-1529.

Parkinson, B. (2009). What holds emotions together? Meaning and response coordination. Cognitive Systems Research, 10, 31-47.

Parkinson, B. (2013). Comment: Journeys to the center of emotion. Emotion Review, 5, 180-184.

Parkinson, B., Phiri, N., \& Simons, G. (2012). Bursting with anxiety: Adult social referencing in an interpersonal Balloon Analogue Risk Task (BART). Emotion, 12, 817-826. 
Parkinson, B., \& Simons, G. (2009). Affecting others: Social appraisal and emotion contagion in everyday decision-making. Personality and Social Psychology Bulletin, 35, 1071-1084.

Reddy, V. (2000). Coyness in early infancy. Developmental Science, 3, 186-192.

Roseman, I. J., Wiest, C., \& Swartz, T. S. (1994). Phenomenology, behaviors, and goals differentiate discrete emotions. Journal of Personality and Social Psychology, 67, 206221.

Schachter, S. (1964). The interaction of cognitive and physiological determinants of emotional state. In L. Festinger (Ed.), Advances in experimental social psychology (Vol 1, pp. 4980). New York: Academic Press.

Snyder, M. (1984). When belief creates reality. In L. Festinger (Ed.), Advances in experimental social psychology (Vol 18, pp. 247-305). New York: Academic Press.

Sorce, J. F., Emde, R. N., Campos, J., \& Klinnert, M. D. (1985). Maternal emotional signaling: Its effect on the visual cliff behavior of 1 year olds. Developmental Psychology, 21, 195200. 